\title{
La investigación de los movimientos sociales desde la sociología y la ciencia política. Una propuesta de aproximación teórica
}

\author{
Josep Pont Vidal \\ Universitat Autònoma de Barcelona. Facultat de C iències Polítiques i Sociologia \\ 08193 Bellaterra (Barcelona). Spain
}

\section{Resumen}

El presente artículo constituye un intento de plantear los principales problemas que representa el estudio de los movimientos sociales desde el punto de vista sociológico y politológico. El artículo tiene un doble objetivo: por un lado, ofrecer una síntesis de los recientes puntos de vista teóricos de la sociología y la ciencia política respecto al estudio de los movimientos sociales. Por otro lado, partiendo de un catálogo de hipótesis y cuestiones, se propone un marco de síntesis teórico en el que pueden inscribirse futuras investigaciones. Finalmente, y en forma de epílogo, se realiza una breve aproximación comparativa sobre la docencia de la acción colectiva y los movimientos sociales en las facultades de sociología en dos países.

Palabras clave: movimientos sociales, asociaciones, grupos de interés, movimientos políticos, «pro-teoría», acción colectiva, docencia de los movimientos sociales, Policy N etworks.

\begin{abstract}
This article attempts to expound the main problems entailed in the study of social movements from the point of view of sociology and political science. The article has a two-fold objective: on the one hand, it offers a synthesis of the recent theoretical points of view found in sociology and political science regarding the study of social movements. $\mathrm{O} n$ the other hand, starting out from a catal ogue of hypotheses and staded questions, a frame work of theoretical synthesis is proposed upon which future investigation can be based. Finally, as an epilogue, there is a brief comparison of the teaching and the investigation of collective action and social movements to be found in D epartaments and Faculties of Sociology in a number of countries.
\end{abstract}

Key words: Social movements, associations, Interest groups, political movements, «Protheory», collective action, teaching and investigation of social movements, Policy N etworks.

\section{Sumario}

1. Breve balance de la investigación de los movimientos sociales

2. Teoría sociológica y movimientos sociales

3. Ciencia política y movimientos sociales
4. Apéndice: la ordenación académica

5. A modo de conclusión

Bibliografía 
El tema de los movimientos sociales se ha constituido en los últimos años como un campo de investigación propio, tanto en las ciencias políticas como en la sociología. Sin embargo, la forma como ha sido abordada la investigación y el tipo de investigaciones realizadas varía de una ciencia a otra.

D esde la sociología, podemos afirmar que ninguna de las actuales corrientes o «escuelas» sociológicas tiene un papel predominante en la elaboración teórica de la investigación de los movimientos sociales. En la investigación de los movimientos sociales se reflejan las mismas dicotomías que en la teoría sociológica, encontrándose a menudo confrontadas entre ellas.

D esde la ciencia política, la situación de la investigación de los movimientos sociales en Europa, a diferencia de losEE.UU ., es un campo relativamente joven. En países como Alemania, donde la investigación de los movimientos sociales se ha constituido como un campo de investigación sociológico desde hace aproximadamente dos décadas, la investigación de este fenómeno desde las ciencias políticas ha sido tradicionalmente enfocado desde perspectivas como la «investigación de los grupos de interés» 0 como «sistemas de mediación política de intereses». Esta situación obedece - como veremos- a determinados enfoques de la historia reciente de la investigación de los movimientos sociales.

Las últimas teorías de la racional choice y sus variantes posteriores y aportaciones de carácter más ideológico han complementado, desde la perspectiva de los intereses colectivos y las dinámicas comunicativas, la investigación desde la ciencia política de los movimientos sociales.

En España, y por razones obvias, la investigación sociológica y politológica reaparece en todas sus especialidades a final es de la década de los años setenta, consolidándose plenamente una década más tarde. Exceptuando casos aislados ${ }^{1}$, la investigación de los movimientos sociales como campo de investigación de la sociología y la ciencia política apenas ha empezado, remontándose, en el mejor de los casos, a principios de los años noventa2. Veinte años después del proceso de instauración del sistema político democrático, si hace-

1. Como una de los primeras investigaciones hay que mencionar los estudios de M. C astells sobre las luchas vecinales en M adrid y Barcel ona durante la década de los años setenta, así como a J. Borja sobre las asociaciones de vecinos en Barcelona. Sobre el movimiento ecologista y antinuclear merece especial atención la investigación de carácter descriptivo realizada por L. Lemkow en 1984. M ás recientemente encontramos el estudio de E. Laraña sobre las movilizaciones estudiantiles a mediados de 1986.

2. Existen numerosas referencias principalmente sobre el movimiento por la paz, el movimiento feminista y el movimiento ecologista de la década de los años ochenta. Sin embargo, se trata en la mayoría de ocasiones de artículos de carácter de análisis político o descriptivo, faltando investigaciones sistemáticas de carácter empírico.

En los años noventa aparecen los primeros estudios empíricos: T EJERIN A, B.; So BRADo, J.M.; AIERDI, X. (1995). Sociedad civil, protesta y movimientos sociales en el País Vasco. Los límites de la teoría de la movilización de recursos. Sobre el movimiento por la paz: VAL CID, Consuelo del (1996). O pinión pública y opinión publicada. Los españoles y el referéndum de la OTAN. M adrid: CIS; PONT, J. (1998). M oviments social a Europa. D el moviment per la pau a les O ngs. Barcelona: H acer; LARAÑA, E. (1999). La construcción de los movimientos sociales. M adrid: Alianza Editorial. 
mos un «balance» de la investigación de los movimientos sociales y de los llamados «nuevos movimientos sociales» - a partir de ahora N M S-, podemos afirmar que - salvo los numerosos estudios realizados por el profesor de psicología E. Laraña, basados especialmente en el concepto de «identidad colectiva», o salvo los estudios empíricos sobre la participación política de Ramón Adell- nos encontramos todavía lejos, tanto a nivel cuantitativo como a nivel cualitativo, de la investigación de otros países europeos. Este déficit lo encontramos en los enunciados teóricos, en los estudios sistemáticos empíricos y en los mismos métodos de análisis empleados. No cabe duda de que esta situación se debe en gran parte al hecho de que la investigación de los movimientos sociales es todavía un campo muy joven en España, a diferencia de países como Francia o Italia, donde el campo de investigación de los movimientos sociales ha alcanzado un considerable nivel.

La presente reflexión constituye un intento de plantear los principales problemas que representa el estudio de los movimientos sociales desde la sociología y la ciencia política ${ }^{3}$. Tiene como objetivos: primero, ofrecer desde la sociología una síntesis teórica que comprenda diferentes niveles teóricos de análisisy, segundo, desde la ciencia política realizar un breve análisis sobre la evolución de la investigación de los movimientos, fuertemente influenciada por elementos históricos, y los puntos de vista de las policy networks4.

D esde la perspectiva de la sociología en la investigación de los movimientos sociales me baso en el modelo analítico y teórico «pro teoría de la desigualdad social y de la acción colectiva» elaborado por el científico social Veit-M ichael Bader5.

D esde la ciencia política, se realiza una aproximación a la investigación de los movimientos sociales partiendo de los caminos y temas en que se ha centrado principalmente el análisis y que han determinado la investigación hasta hace pocos años. Parto básicamente de los estudios del politólogo alemán 0 ssip Flechtheim, como pionero en la ciencia política al abordar la relación entre movimientos sociales, partidos políticos y grupos de interés en las modernas

3. En un nivel más amplio, F. J. Llera Ramo, constata las «interferencias» existentes entre la ciencia política y la sociología. El autor aboga por la sociología política como un intento para responder a la cuestión planteada. Entre las circunstancias de tal es interferencias, menciona las siguientes:

1. La compresividad y la amplitud de las implicaciones de sus respectivos objetos (la política y la sociedad).

2. La pretensión imperialista de la sociología desde sus orígenes comtianos.

3. El más temprano desarrollo de ésta como disciplina científica.

4. La mayor dependencia inicial de la ciencia política respecto de las transformaciones del Estado democrático.

5. La afirmación de la ciencia política como disciplina científica en un campo y desde un enfoque y una metodología.

LleRA RAM O, F.J. (1996). «Ciencia política y sociología política: la necesaria reconstrucción de la interdisciplinariedad». Reis, 76.

4. Entre otros cabe destacar el estudio de Bernd M ARIN y Renate M AYNTZ, Policy N etworks:

Empirical Evidence and theoretical Considerations.

5. Veit-M ichael BADER. Protheorie sozialer Ungleichheit und kollektiven $\mathrm{H}$ andels. 
sociedades democráticas. A partir de las mencionadas aproximaciones teóricas, se expone un catálogo de hipótesis y cuestiones en el que pueden inscribirse futuras investigaciones. Finalmente, como apéndice, y con el propósito de animar a la institucionalización del estudio del fenómeno en un país tan cargado de experiencias derivadas de la acción colectiva como es España, el escrito se complementa con la ordenación académica de la sociología de la acción colectiva y los movimientos sociales en dos facultades de sociología: Facultad de Sociología de la U niversidad de Bielefeld (Alemania) y la Facultad de Sociología de la U niversidad de M assachusetts - campus de Amherst- (EE.UU .).

\section{B reve balance de la investigación de los movimientos sociales}

Las ciencias sociales se ocupan de la complejidad del fenómeno de los movimientos sociales desde hace aproximadamente ciento cincuenta años, con la aparición de los primeros estudios derivados de los cambios sociales producidos por la revolución industrial, al ser los movimientos sociales un factor importante de cambio social y de desarrollo político. Sin embargo, y salvando las diferencias específicas de cada país, el interés científico sobre los movimientos sociales ha pasado por diferentes fases. En los países de la Europa occidental el estudio del fenómeno de los movimientos sociales - por razones que más adelante veremos- prácticamente desapareció en los años que siguieron la posguerra, no volviendo a despertar interés en las ciencias sociales hasta bien entrada la década de los años setenta.

La investigación de los movimientos sociales abarca desde el análisis del primer movimiento obrero realizado básicamente por Lorenz von Stein en Alemania, hasta trabajos politológicos aparecidos a principios de la década de los años sesenta. Sin embargo, es a comienzos del siglo Xx cuando se producen las primeras investigaciones de Ferdinand Tonnies, Rudolf $\mathrm{H}$ aberle y M arianne y $M$ ax Weber. En los países europeos - especialmente en Alemania- , los movimientos sociales han sido estudiados partiendo de tres caminos diferentes: como una parte descriptiva de la historia de las ideas, como una parte de la movilización política en los sistemas totalitarios, o bien centrándose en el movimiento obrero. En España la investigación de los movimientos sociales es de carácter sociohistórico y descripitivo, centrándose básicamente en el movimiento obrero y anarcosindicalista de los anteriores años de la G uerra C ivil, siendo los investigadores sociales comprometidos con los movimientos ${ }^{6}$. Aunque el concepto de movimiento social tienen connota-

6. Entre los numerosos estudios cabe mencionar:

LoREnZO, A. (1946-1947). El proletariado militante. Tolouse.

BuEn CASA, M. (1966). El movimiento obrero español (1886-1926). París.

PEIRó, J. (1959). Pensami ento, trayectoria de la CN T. I deas sobre sindicali smo y anarquismo. M éxico.

So u CHY, A. (1937). Entre campesinos de Aragón. El comuni smo libertario en las comarcas liberadas. Barcelona.

Cabe también destacar los exhaustivos estudios realizados por Víctor Alba, sobre el PO U M. 
ciones con el «movimiento nacional», este hecho no ha sido causa - a diferencia de Alemania- de que la investigación sociológica haya estado influenciada por este fenómeno histórico y político. Las primeras investigaciones de la postguerra se producen en losEE.UU . y son llevadas a término por el sociólogo Robert E. Park 7 . En estas investigaciones también hay aportaciones de la psicología de masas realizadas en Francia e Italia. Sin embargo, el estudio de las causas que provocaron el conflicto y la crisis del sistema de participación democrática y la subida al poder de los movimientos fascista y comunista de los años treinta, es la causa que provoca un redescubrimiento del fenómeno de los movimientos sociales como disciplina sociológica central. D el mismo modo, y a raíz de la experiencia mundial de los movimientos totalitarios, los movimientos sociales fueron conceptualizados como un fenómeno potencialmente peligroso de forma de conducta colectiva política en las democracias occidentales.

La obra Social M ovements. An Introduction to Political Socialogy del profesor de sociología R. H eberle (1951) marca un nuevo comienzo del estudio de la ideología y la participación de los individuos en los movimientos sociales. En los años sesenta se desarrolla la perspectiva de la conducta colectiva consolidándose en posteriores escuelas. Por un lado, el interaccionismo simbólico de $H$. Blumer, se centró en las normas emergentes, en los procesos de self regulación y en las normas internas de los individuos. Por otro lado, el funcional estructuralismo de Talcott Parsons, vio en determinantes sociales como las «tensiones» los impulsos de la acción colectiva. D e forma similar, R. Turner y $L$. Killian (1957), basándose en el funcional estructuralismo, consideraron a los movimientos sociales como un fenómeno creativo de cambio. D esde el punto de vista macro, Smelser, en la Theory of Collective Behaviour (1962) vio en la acción colectiva la respuesta colectiva frente a las tensiones de la sociedad.

D esde el marxismo, se ha interpretado a los movimientos sociales con expectación y reservas: por un lado, como fenómeno colectivo para impedir el colapso del capitalismo y, por otro lado, como fenómeno de movilización para ser sustituido por el socialismo. Para los marxistas, los movimientos sociales son expresiones colectivas de descontento social y de cambio social.

Los debates actuales se centran en la «racionalidad» de los fenómenos colectivos, en los estudios de la movilización de recursos, en los mecanismos por los que los movimientos sociales reclutan a nuevos miembros y en las formas en que la movilización ciudadana y la movilización de recursos tienen un papel decisivo en los movimientos.

En España, a pesar de las numerosas acciones masivas de protesta colectiva y de participación política no convencional en las dos últimas décadas, el campo de investigación de los movimientos sociales apenas se ha iniciado

7. La tesis doctoral de Robert E. Park: M asse und Publikum. Eine methodologi sche uns sozi ologische U ntersuchung, defendida en 1904, no fue un examen sobre el discurso de la psicología de masas del siglo XIx, sino que sentó las bases para el discurso de masas desarrollado posteriormente por él mismo y por científicos sociales como Tarde y Le Bon. 
como campo de investigación de la sociología y la ciencia política. La investigación realizada, a semejanza de otros países europeos como Francia, Italia - Alemania, el análisis del contexto político y la transformación histórica de los movimientos, ha sido determinante en la orientación de los estudios ${ }^{8}$. A comienzos de los años ochenta, se han centrado principalmente en el movimiento obrero ${ }^{9}$, y su conexión con el movimiento cristiano ${ }^{10}$, o en el movimiento vecinal11 o sobre los movimientos sociales ${ }^{12}$, durante los primeros años de la transición democrática. En los últimos años, se han realizado investigaciones que se basan en los puntos de vista teóricos elaborados por Touraine, O ffe, M elucci y J. Guafield. Sin embargo, siguen siendo escasas las investigaciones de carácter empírico, que nos permitan desarrollar un marco teórico de acuerdo con las peculiares características de las identidades colectivas, las identidades regionales y la modernización. Entre los escasos estudios hay que destacar los realizados sobre las movilizaciones estudiantiles de los años 1986 y 1988, por E. Laraña; cabe también mencionar los estudios analíticos sobre las movilizaciones y la participación de finales de los años setenta y principios de los ochenta, realizados por Ramón Adell.

8. La influencia de J. Galtung, C. O ffe y del profesor de sociología de la Universidad de California Joseph Gusfield, ha sido muy extendida en la mayoría de ellos. Entre los estudios sobre movimientos social es y el contexto político del postfranquismo cabe mencionar: Álvarez Jun CO, José. «M ovimientos sociales en España: del modelo tradicional a la modernidad postfranquista». En LARAÑ A, E.; GUSFIELD, J. (1994). Los nuevos movimientos sociales. M adrid, p. 413-443.

PASTO R, Jaime (1992). «M ovimientos sociales y acción colectiva». Ponencia presentada en el IV C ongreso Español de Sociología. M adrid, 1992.

9. Existe una extensa bibliografía al respecto. La comunicación que se menciona a continuación ofrece un detallado estudio de las publicaciones sobre el movimiento obrero en España. En Estivill, J.; T OMȦs, J.; C ARRAsq Uer, P.; T ORN S, T. (1985). «La sociologia del treball a Catal unya (1974-1980)». Actes de les primeres jornades catal anes de sociol ogia (1981). Entre otras obras cabe destacar: TEZAN o S, J.M . (1982). ¿Crisis de la conciencia obrera?; LU DEVID, M. El movimiento obrero en Cataluña bajo el franquismo; CLAUdín, F. El nuevo movimiento obrero español; FISH M AN , R. (1984). «EI movimiento obrero en la transición: objetivos políticos y organizativos. Reis, p. 61-112.

10. Entre los estudios más recientes ver: Francisco FERn Án DEZ BuEY, «N uevos movimientos sociales, izquierda alternativa y cuestión cristiana». AA.VV. (1991). Euroizquierda y cristianismo: presente y futuro de un diálogo; C O STA, Joan (1997). D el s moviments d'E sglésia a la militància política.

11. Entre los estudios realizados hay que mencionar:

VILLASANTE, T. (1976). Los vecinos en la calle.

- (1991). M ovimientos ciudadanos e iniciativas populares.

D esde una perspectiva descriptiva e histórica: H UERTAS, J.M .; AN D REU, M . (1996). Barcelona en Iluita (El moviment urbà 1965-1996). H ay que mencionar también las investigaciones realizadas por Anna Alabart.

12. Pérez Agote, Alfonso (1987). «El nacionalismo radical vasco. M ecanismos sociales de su aparición y desarrollo». Trabajo presentado en el seminario Los movimientos sociales y el fin de las ideologías. U niversidad Internacional M enéndez Pelayo, Santander; Tejerin A, B.; So brad 0, J.M.; AIERDI, X. (1995). Sociedad civil, protesta y movimientos sociales en el País Vasco. L os límites de la teoría de la movilización de recursos. Vitoria-G asteiz: Servicio C entral de Publicaciones del Gobierno Vasco. 


\section{Teoría sociológica y movimientos sociales}

El estudio y la observación de los fenómenos sociales es muy complejo y varía según el país, la región o el círculo cultural que se investigue. Factores como el origen, el desarrollo, los cambios producidos o el éxito y la transformación de los movimientos sociales varía de un país a otro. Todo estudio que se realice desde el punto de vista teórico ha de tener en cuenta los mencionados factores.

La investigación actual de los movimientos sociales se encuentra con las mismas contradicciones y callejones sin salida en que se pueda encontrar la investigación de la acción colectiva, la investigación de la participación política o la investigación de los grupos de interés. Esta situación la encontramos tanto a nivel conceptual, como a nivel teórico o empírico.

A nivel conceptual, encontramos definiciones contradictorias entre sí, poco claras y en muchos casos ambiguas. Tanto en las diferentes corrientes y puntos de vista teóricos de las ciencias sociales como en la ciencia política, existen grandes ambigüedades a la hora de definir qué se entiende por movimiento social, protesta colectiva, organización, identidad colectiva, actores colectivos, etc.

A nivel teórico, los puntos de vista como el «social psicológico» se encuentran confrontados con las «teorías macrosociológicas», las «teorías de la estructura» se encuentran confrontadas con las «teorías del actor» y aś las teorías de la «privación relativa» con las «teorías de la identidad», las «teorías de la movilización de recursos», teorías «culturalistas» con la teoría «economicista», teorías de la «policy approach» con las teorías «micro», «meso», «macro». A ello deberíamos añadir que los mencionados puntos de vista teóricos todavía no han sido suficientemente analizados por posteriores trabajos empíricos.

Por lo que se refiere a la investigación empírica de los movimientos sociales, existen todavía problemas implícitos derivados de la misma desconfianza existente entre la teoría sociológica y la investigación y la metodología empírica, y en la profunda división entre construcción teórica y experiencia. En muchas ocasiones los resultados empíricos se han utilizado acríticamente para legitimizar determinadas teorías, relevando a un segundo plano los resultados que no encajaban con las hipótesis teóricas. Sin embargo, también desde el punto de vista teórico, han aparecido numerosas lagunas al faltar a estos enunciados una comprobación empírica, quedando en generalizaciones y abstracciones apenas comprobables empíricamente.

L legados a este punto, podemos preguntarnos qué estrategia de análisis se propone. La exigencia para superar la dicotomía teórica no es nueva, ya que la encontramos en diferentes puntos de vista teóricos. $C$ abe señalar que no es una tarea sencilla encontrar un punto de partida común entre diferentes teorías en "concurrencia», la tarea, pues, ha de tener como punto de partida el análisis de los diferentes elementos cognitivos procedentes de diferentes puntos de vista teóricos más o menos consistentes. Con ello no se trata de realizar una ecléctica integración de teorías, sino más bien una integración de diferentes perspectivas y conocimientos. Así pues, parto de la «pro-teoría» pro- 
puesta por V. M. Bader13, cuyo modelo de la acción colectiva se basa en las siguientes tesis:

1. Continuidad de la acción colectiva cotidiana y conflictos colectivos. Siguiendo los postulados de M arx y Turner y la crítica implícita a las posiciones dominantes de la psicología social, se tematiza la acción colectiva no institucional como un fenómeno social.

2. La acción colectiva se orienta de forma racional y estratégica. En conexión con las theories of rational choice se parte de la racionalidad de la acción colectiva, pero a diferencia de ésta, se desarrolla un amplio marco conceptual en el que no se reduce la racionalidad solamente a nivel cognitivo 0 estratégico.

3. La integración de las diferencias sociales y la acción colectiva. La separación fáctica entre la investigación de las desigual dades y la investigación de los movimientos sociales es uno de los puntos débiles de los actuales deba tes. En conexión con los puntos teóricos de G iddens y Bourdieu, la teoría de la estructuración de la acción permite sobrepasar los sistemas confrontados tradicionales como los puntos de vista del actor versus sistema o del estructuralismo-determinismo versus accionismo-voluntarismo. Un punto de vista teórico sobre movimientos social es ha de incluir el ementos como las condiciones estructurales en que se desarrollan, sobre la formación y sobre la movilización de los grupos en conflicto.

4. La integración de las condiciones económicas, culturales y políticas. Los movimientos sociales no pueden ser explicados exclusivamente desde el punto de vista materialista o economicista. Éstos se orientan hacia las normas y los modelos dominantes.

5. La integración del concepto de habitus, identidad colectiva y movilización de recursos. No es suficiente creer que a partir de la investigación ahistórica de la actitud pueden ser explicados aspectos como la identidad colectiva, la cultura o la acción colectiva. A la inversa, tampoco es suficiente creer que a partir del estudio de las normas, formas alternativas y identidades alternativas o mundos de vida - en el sentido de J. H abermas- pueden ser explicados los movimientos sociales.

6. La transmisión de objetivos y condiciones subjetivas de la acción colectiva. Las investigaciones de los movimientos social es han de tener en cuenta las condiciones objetivas y las causas estructural es de la acción colectiva.

7. La conexión de los modelos lineal es con modelos de reacoplamiento y modelos estratégicos de intersección.

13. Kollektives $\mathrm{H}$ andeln. Protheorie sozialer U ngl eichheit und kollektiven $\mathrm{H}$ andels. En forma resumida, su obra parte del siguiente punto de vista: construye un marco de referencia suficientemente complejo en el cual se muestra un modelo de los diferentes niveles de análisis de la acción colectiva y de los movimientos sociales. La estrategia utilizada por Bader se basa, por un lado, en la disgregación conceptual y, por otro, en una diferenciación de niveles de análisis con sus respectivos contenidos teóricos. 
Una de las dificultades centrales a la hora de elaborar una teoría de la acción colectiva es el desarrollo de los modelos complejos de interacción. En la pro teoría se muestra el desarrollo de la acción colectiva, los movimientos sociales y los conflictos a diferentes niveles. En cada uno de estos niveles existen sin embargo ámbitos amplios y alternativos de análisis.

Como principales cuestiones para las futuras investigaciones habría que tener en cuenta:

- ¿Podemos hablar de la existencia estructural de una nueva clase media como principal actor de los movimientos sociales?

- ¿Los movimientos sociales, tienen unas finalidades y representan unos valores nuevos y homogéneos?

- ¿Están los movimientos sociales constituidos cualitativamente por nuevas formas de vida y nuevas identidades?

- ¿Se manifiestan nuevas formas organizativas?

- ¿Se trata de movimientos uniformes o más bien de movimientos que forman parte de un proyecto global?

- ¿Representan nuevos modelos cognitivos y significados, como «ideologías» $0 \ll$ «alores»?

\section{Ciencia política y movimientos sociales}

En la ciencia política el concepto de movimiento social ha estado íntimamente vinculado con los conceptos «asociación» y «grupos de interés», y ha sido estudiado a partir de la década de los años cincuenta. D entro de la tradición del estatismo y del estudio de la naturaleza y existencia de las asociaciones y grupos de interés; se han visto a los grupos de interés como un peligro para la completa soberanía del Estado. Entre éstos, el politólogo alemán T heodor Erschenburg14 dijo que los «grupos de intereses egoístas» de las asociaciones, deslegitimizan la «autoridad del Estado», a la vez que socavan las condiciones necesarias para el consenso político de los sistemas democráticos. Erechenburg contrapone a ello un cuerpo de funcionarios independientes que pueda tomar decisiones técnicas adecuadas. En este modelo no habría cabida para los movimientos sociales dentro del sistema político.

D es de la perspectiva de las teorías del pluralismo han aparecido críticas a este modelo. Los defensores de la visión del pluralismo proponen la toma de la decisión política adecuada, preguntándose a su vez sobre las condiciones en que tienen lugar en las democracias modernas. La democracia de masas deja al individuo en un rol y situación totalmente impotente, teniendo solamente dos opciones para su integración política: identificarse con un sistema de dominación totalitario o asumir el val or de participar en la sociedad - civil 
society- , dividida a su vez en diferentes grupos de interés. En este sentido, el Parlamento, los partidos políticos y la opinión pública, los cuales tienen a su vez el origen en el sistema liberal del siglo XIX. Las modernas democracias no son suficientes para la integración política en la sociedad de los medios tradicionales para resolver las nuevas exigencias y problemas planteados. La combinación ideal sería la superación del pluralismo tradicional del sistema libe ral para desembocar en una red en concurrencia de diferentes grupos de interés. En este sentido, los partidos políticos tendrían jurídicamente la misma capacidad de intervención que otros grupos de interés. Las asociaciones tendrían una función catalizadora. La teoría del pluralismo como parte del movimiento obrero del período de entreguerras habría sido válida en diferentes democracias europeas, especialmente en la Alemania de la República de Weimar, al formar parte de la historia de las ideas del movimiento obrero.

Con la transformación de intereses del movimiento obrero en el sistema político electoral de las instituciones modernas, se perdieron determinados aspectos importantes para dicho movimiento obrero. Al igual que en el campo de la sociología, la experiencia del nacional socialismo determinó la postura de la politología respecto a los movimientos sociales.

A partir de esta experiencia, los estudios se centraron en el sistema de las asociaciones. Sin embargo, al igual que los movimientos sociales, tiene que cumplir determinadas condiciones con el sistema democrático. También es válido para el caso de las asociaciones. Según Fraenkel15, los grupos de interés tendrían que allanar, en definitiva, el camino para las decisiones políticas.

Investigaciones realizadas durante la década de los años ochenta concedieron una enorme importancia a los grupos de interés en su relación con los N M S. En este sentido, hay que diferenciar entre estos dos fenómenos de acción colectiva. Al examinar el fenómeno de los N M S vemos que éstos no son grupos organizados de forma jerárquica, como tampoco tienen a los partidos políticos y al Parlamento como sus destinatarios principales. El politólogo O tto Stammer concede al término «movimiento social» un papel clave en el cambio social - de forma muy parecida que R. H eberle-, «como cambio fundamental del orden social» (Stammer, 1955: 305) y clasifica y relaciona los movimientos sociales en dos contextos diferenciados: por un lado, en estrecha relación con el movimiento socialista y, por otro lado, con la llamada «inves-

15. Según Fraenkel, las asociaciones han de cumplir ciertos requisitos: a) estar suficientemente organizadas y tener suficiente poder para poder dirigir las actividades de sus miembros; b) poseer estructuras transparentes y democráticas, además de estar supeditadas a las demandas plebiscitarias de la población; c) tener una limitación en las gratificaciones particulares, además de estar orientadas al bien común, especialmente los sindicatos; d) tener unos condicionamientos limitados para su participación en el sistema parlamentario.

FRAEN KEL, E. (1958). «D ie repräsentative und die plebiszitäre K omponente im demokratischen Verfassungstaat». En D ers. D eutschland und die westlichen D emokratien. Stuttgard, p. 113-151.

- (1960). «D eutschland und die westlichen D emokratien». En D ers. D eutschland und die westlichen D emokratien. Stuttgard, p. 32-47. 
tigación del totalitarismo». Los «partidos democrático-socialistas» están vinculados con los movimientos sociales a nivel «de ideales y a nivel personal», teniendo, a diferencia del resto de partidos políticos, mucho más que «unos fines políticos limitados». Esta función atribuida a los movimientos sociales modernos tendrá consecuencias en los movimientos totalitarios y en la investigación del totalitarismo. Por otro lado, por lo que se refiere al estudio de los actuales movimientos sociales, vincula el cambio estructural de las democracias occidentales con la investigación sobre el total itarismo: «la finalidad de la politización de la sociedad con la ayuda de un sistema de planificación de las acciones sociopolíticas que, según R. H eberle, caracteriza como sociedades impulsadas por los social movements y abarca todos los campos del dominio».

A partir de finales de la década de los años cincuenta, aparecen movimientos de masas espontáneos - como la revuelta de H ungría en 1956 y la aparición de «consejos»- que no pueden ser explicados desde la investigación del totalitarismo. O ssip K. Flechtheim, en su obra Fundamentals of Political Science, ya propone, unos años antes, nuevos caminos de análisis y de interpretación. Aunque de sus tesis se deduce un acentuado pesimismo por lo que se refiere a los partidos populares, el autor diferencia claramente entre dos grupos de interés: las asociaciones o pressure groups y los movimientos políticos. Según Flechtheim, las asociaciones son organizaciones que persiguen un interés particular, creando un clima de opinión favorable para imponer su política a los partidos políticos. Un elemento típico de los grupos de presión sería la «imitación en sus finalidades». Como consecuencia de esta limitación, el sistema y la red de asociaciones sería incapaz de poder influenciar de forma adecuada frente a los problemas políticos. Las asociaciones, en lugar de impulsar el bien común, constituyen un «sistema obligado monopolístico y burocrático», no buscando la solución de problemas ni la democratización de la sociedad. En este contexto, los movimientos sociales constituyen la alternativa. Los movimientos políticos disponen dentro del marco democrático de una función innovadora. Así, mientras que los grupos de interés se centran de forma limitada en sus finalidades particulares, los movimientos políticos tendrían una finalidad mucho más amplia, dirigiendo su acción hacia los mismos principios y estructuras sociales para destruirlas o modificarlas. Un ejemplo de movimientos políticos son los movimientos de liberación o los movimientos de clase - como el movimiento obrero- , siendo la acción política todo tipo de desobediencia civil.

Sin entrar en la génesis del concepto de democracia o en su posible validez, sí que podemos afirmar que el trabajo del politólogo Fechtheim ha contribuido decisivamente a que determinados aspectos de los N M S sean objeto de atención y estudio científico, pudiendo ser considerado como el fundador de los estudios de los N M S desde las ciencias políticas.

En los últimos años se han desarrollado nuevos modelos interpretativos para el estudio de los movimientos sociales - como actores colectivos- al ser estos un factor clave para el surgimiento y la consolidación de las políticas sociales. 
Las teorías de la elección racional han utilizado técnicas matemáticas para predecir las posibilidades de éxito de los movimientos sociales. Sin embargo, no han tenido en cuenta los aspectos ideológicos, culturales, o el mismo habitus de los actores.

Un punto de vista más desarrollado, las policy networks, nos ofrece un marco instrumental de análisis para la descripción de las formas de comunicación y de interacción en las sociedades complejas, a partir de perspectivas empíricas. Para el anál isis de los actores col ectivos y los movimientos sociales, se ofrecen diferentes criterios analíticos, como el nivel de institucionalización, el nivel de centralización, la exclusividad o el monopolio de su campo de acción, el grado de estabilidad, etc.

\section{Apéndice: la ordenación académica}

El fenómeno de los movimientos sociales se ha establecido en las ciencias sociales como un campo independiente de investigación. En la ciencia política el fenómeno se estudia básicamente en conexión con los grupos de interés.

El desarrollo de la investigación de los movimientos y la implantación académica varía según el país. Sin la pretensión de querer profundizar en el proceso de consolidación de la investigación en otras países, citaré solamente los ejemplos de las facultades de sociología de la U niversidad de Bielefeld (Alemania) y universidad norteamericana de M assachusetts.

En la Facultad de Sociología de la U niversidad de Bielefeld el tema de los movimientos sociales aparece regularmente como asignatura de libre el ección. Los seminarios se inscriben dentro del área de la teoría política y se centran en la «Teoría de los movimientos sociales» 0 en la «H istoria y sociología de los movimentos de resistencia».

En los EE.UU ., los movimientos social es ocupan un lugar clave en la vida política del país 6 . De esta forma, la investigación y la oferta académica constituyen un factor determinante en la licenciatura de Ciencia Política y Sociología. El caso de la Facultad de Sociología de la U niversidad de M assachusetts - campus de Amherst - constituye un ejemplo. En esta Facultad, en el curso académico 1997-1998, se ofrecen cinco asignaturas que tratan de una forma u otra el tema de los movimientos sociales sobre un total de treinta y siete. D os asignaturas Ilevan como título «M ovimientos sociales». En ellas se analiza el fenómeno desde la perspectiva del análisis de la estruc-

16. En los EE.UU ., los movimientos sociales, los movimientos políticos y los grupos de interés tienen un papel clave en la vida política del país. Entre los principales movimientos sociales y políticos cabe destacar los movimientos por los derechos civiles, los movimientos de los grupos homosexual es o el Ilamado movimiento «9 to 5» de los empleados de oficinas. Entre la extensa bibliografía podemos mencionar: AA.VV. (1983). D ollars \& Träume. Studien zu Politik. Ö konomie und Kultur der USA. H amburg.

Rim m erman , Craig (1997). T he N ew Citizenship. Unconventional Politics, Activism, and service.

SH ABECOFF, Phillip (1993). A Fierce Green Fire: The American Environmental M ovement. 
tura, significación e ideología, incluyendo el estudio de casos concretos. El tema «Acción colectiva» constituye otras dos asignaturas. El enfoque parte de los procesos de acción colectiva y de los movimientos sociales como elementos de cambio social. Finalmente, dentro del seminario de «Sociología política», el tema de los movimientos sociales es analizado desde la perspectiva comparada de las sociedades democráticas y totalitarias y los movimientos extremistas y totalitarios.

En España todavía no se ha establecido definitivamente como una disciplina, ni ha producido hasta la fecha suficientes estudios de carácter empírico que nos permitan analizar el fenómeno desde las características propias de nuestro país 17 , al tratar algunas publicaciones recientes de puntos de vista teóricos de otros países, principalmente Alemania, Francia e Italia.

A nivel académico, existen todavía importantes lagunas cuanto a la oferta de docencia ofrecida principalmente en las respectivas facultades de ciencia política y sociologia y de la consolidación de grupos de investigación sobre el tema. Es evidente que muchas facultades son todavía de reciente creación, y que algunas de ellas están todavía en fase de consolidación. Sin embargo, y dada la importancia sociológica y politológica de la acción colectiva y la participación política no convencional, sigue siendo la «asignatura pendiente».

\section{A modo de conclusión}

La investigación de los movimientos sociales ha tenido diferentes fases históricamente determinadas. En la actualidad ya no se centra exclusivamente en la historia de las ideas, en la movilización política de los sistemas totalitarios o en el movimiento obrero. D esde la ciencia política y la sociología, Ios puntos de vista teóricos de las policy networks y la «pro-teoría» constituyen unos intentos altamente desarrollados que nos permiten, desde diferentes ciencias, analizar el fenómeno de los movimientos sociales en las complejas sociedades actuales.

El punto de vista teórico de la «Pro-teoría» es un intento de superar las dicotomías existentes. La acción colectiva se orienta de forma racional y estratégica, pero los movimientos sociales no pueden ser explicados exclusivamentea partir de presupuestos positivistas y de la acción egoísta y individual ista. Para su estudio hay que integrar, por un lado, las condiciones económicas, culturales y políticas y, por otro lado, el concepto de habitus, identidad colectiva y la movilización de recursos.

Los puntos de vista teóricos de las policy networks, nos permiten superar los modelos teóricos formales excesivamente centrados en la elección racional, los cuales no tienen en cuenta elementos como la tradición socialista y emancipatoria de los movimientos sociales.

17. Tejerin A, B.; So brad o , J.M .; Aierdi, X. (1995). Soci edad ci vil, protesta y movimientos sociales en el País Vasco. Los límites de la teoría de la movilización de recursos. Vitoria-G asteiz: Servicio central de publicaciones del Gobierno Vasco. 
Finalmente, cabe mencionar los escasos estudios sobre acción colectiva y movimientos sociales que se realizan en España, así como su incipiente oferta académica. Como impulso de partida recientes congresos apuntan a una cierta normalización en el futuro's.

A nivel académico, existen todavía lagunas en cuanto a la oferta de docencia en las respectivas facultades de Ciencias Políticas y Sociología y de la consolidación de grupos de investigación sobre el tema ${ }^{19}$. Es evidente que muchas Facultades son todavía de reciente creación, y que al gunas de ellas están todavía en fase de consolidación. La acción colectiva y la participación colectiva no convencional siguen siendo, de todas formas, la «asignatura pendiente».

\section{Bibliografía}

Álvarez Jun co, José (1994). «M ovimientos sociales en España: del modelo tradicional a la modernidad postfranquista». En LARAÑ A, E.; G USFIELD, J. (1994). LoS nuevos movimientos sociales. M adrid, p. 413-443.

ADELL, Ramon (1993). «M ovimientos sociales y participación política». Revista de Estudios Políticos, 82. O ctubre-diciembre de 1993, p. 177-194.

- (1998). «El estudio de la movilización como indicador de participación sociopolítica: parámetros analíticos y significados», ponencia del VI C ongreso Español de Sociología, La Coruña, 25 de septiembre de 1998.

BADER, Veit-M ichael (1991). Kollektives H andeln. Protheorie sozialer U nglei chheit und Kollektiven $\mathrm{H}$ andelns. Teil 2. O pladen.

BAder, Veit-M ichael; Bensch op, A. (1989). Ungleichheiten. Protheorie sozialer Ungleichheit und kollektiven $\mathrm{H}$ andelns. Teil 1 . O pladen.

C OSTA, Joan (1997). D els moviments d'E sglésia a la militància política. Barcelona:

Ed. M editerrània.

BEYM E, Klaus von (1980). Interessengruppen in der Demokratie. M unich.

ELSTER, J on (1990). «R acionalidad, moralidad y acción colectiva». Z ona abierta, núm. 54-55, p. 43-68.

ERSCHENBURG, Theodor. (1955). H errschaft der Verbände?

Evans, R. (ed.) (1969). Readings in Collective Behavoir. Chicago: Rand M cN ally.

Eyerm An, Ron; Jam ISO N, Andrew (1991). Social M ovements. A Cognitive Approach.

Pennsylvania State University Press.

FraEN KEL, Ernst (1958). «D ie repräsentative und die plebiszitäre Komponente im demokratischen Verfassungstaat». En D ers. D eutschland und die westlichen Demokratien. Stuttgard, p. 113-151.

- (1960). «D eutschland und die westlichen D emokratien». En D ers. D eutschland und diewestlichen D emokratien. Stuttgard, p. 32-47.

18. La celebración del Second European C onference on Social M ovements en la U niversidad del País Vasco (V itoria-G asteiz) durante el mes de octubre de 1996 constituye un paso importante. En esta C onferencia se presentaron recientes trabajos empíricos sobre movimientos sociales, de protesta política y constitución de redes que se realizan actual mente en España.

19. Cabe destacar el grup de investigación dirigido por E. Laraña en la U niversidad Complutense de M adrid. 
FLECH TH EIM , O ssip K. (1952). Grundlegung der Politischen Wissenschaft. M eisenheim am Glan.

- (1962). «Parteien, Bewegungen, Pressure G roups». En: D ers: Zeitgeschichte und Zukunftpolitik. $\mathrm{H}$ amburgo.

H eberLe, Rudolf (1951). Social M ovements: An Introduction to Political Sociology. Lousiana State University.

Larañ A, Enrique (1993). « os movimientos sociales en España». En: Cam Po, Salustiano del (ed.). Tendencias Sociales en la España de hoy. Bilbao.

- (1994). «C ontinuidad y unidad en las nuevas formas de acción colectiva. Un análisis comparado de movimientos estudiantiles». En: LARAÑ A, E.; G U SFIELD , J. (1994). Los nuevos movi mientos sociales. De la ideología a la identidad. M adrid: CIS.

- (1999). La construcción de los movimientos sociales. M adrid: Alianza Editorial.

LEM KOW, Luis (1984). La protesta antinuclear. M adrid: M ezquita.

LUDEVID, M anuel (1977). El movimiento obrero en Cataluña bajo el franquismo. Barcelona.

M ARIN, Bernd; M AYNTZ, Renate (eds.) (1991). Policy N etworks: Empirical Evidence and Theoretical Considerations. Colonia: Campus Verlag.

M o LINA, J. (1994). «Los grupos de interés en España». En: Papeles de la Fundación. FAES.

O fFE, C laus (1988). Partidos políticos y nuevos movimientos sociales. M adrid: Editorial Sistema.

O LSON, M . (1986). Auge y decadencia de las naciones. Barcelona:Ariel.

PARSO N, Talcott (1969). Politics an Social Structure. N ueva York: Free Press.

PASTOR, Jaime (1992). «M ovimientos sociales y acción colectiva». Ponencia presentada en el IV Congreso Español de Sociología. M adrid, 1992.

PONT, J Osep (1997). Condicions socials sobre I 'ori gen dels N ous M oviments Socials. Anàlis comparativa entre la República Federal d'Alemanya i Espanya. Exemple d'estudi: el moviment per la pau. (Tesi doctoral, U niversitat de Barcelona).

- (1997). «El model sistèmic de C. O ffe i J. H abermas. Elements per al'estudi anàlitic i comparatiu dels moviments socials». Perspectiva Social, 39, p. 15-34.

- (1998). M oviments socialsa Europa. D e movmient per la pau a les O N Gs. Barcelona: $\mathrm{H}$ acer.

RASCH KE, Joachim (1988). Soziale Bewegungen. Ein historisch-systematischer G rundriss. Frankfurt/N ueva York: Campus Verlag.

RieCH M AN N , Jorge; FeRn Án deZ BUeY, Francisco (1994). Redes que dan libertad. Introducción a los nuevos movimientos sociales. Barcelona: Paidós.

Rödel, U.; D ubiel, H .; Fran Ken Berg, G . (1989). Die demokratische Frage. Frankfurt a M.

SM ELSER, N eil J. (1963). T heory of Collective Behavior. Trad. en castellano (1989).

Teoria del comportamiento colectivo. M éxico: Fondo de Cultura Económica.

Stam m er, 0 tto (1955). «Politische Soziologie». En Gehlen, Arnolt; SCHelSKY, H elmut (hg.): Soziologíe. Ein Lehrund $\mathrm{H}$ andbuch zur modernen Gesellechaftskunde. Düsseldorf-Köln, p. 277-333.

- (1957). «nteressenverbände und politische Parteien». En D ers. Politische Soziologie und Demokratieforschung. Berlín (1965), p. 116-136. 
Tejerina, Benjamín; So brado, José M anuel; Aierdı, Xabier (1995). Sociedad civil, protesta y movimientos sociales en el País Vasco. Los límites de la teoría de la movilización de recursos. Vitoria-Gasteiz: Servicio Central de Publicaciones del Gobierno Vasco.

TEZAN OS, J.F. (1982). ¿Crisis de la conciencia obrera? M adrid: M ezquita.

VAL CID, Consuelo (1996). O pinión pública y opinión publicada. Los españoles y e referéndum de la OTAN. M adrid: CIS.

VILLASANTE, T.R. (1991). M ovimientos ciudadanos e iniciativas populares. M adrid: HOAC. 